\title{
IMPACT ON DIET QUALITY AND RESILIENCE IN URBAN COMMUNITY DWELLING OBESE WOMEN WITH A NUTRITION AND PHYSICAL ACTIVITY INTERVENTION
}

\author{
A. Taetzsch ${ }^{1}$, D. Quintanilla', S. Maris ${ }^{2}$, J. Letendre', L. Mahler ${ }^{3}$, F. Xü2, M.J. Delmonico², I.E. Lofgren ${ }^{1}$
}

\begin{abstract}
Objective: To examine the effect of a Tai Chi, resistance training, and behaviorally-based diet education intervention on dietary quality as well as resilience and physical resilience in obese older women. Design: Community health outreach with a quasi-experimental design. Setting: An urban senior center in Rhode Island. Participants: Thirty-three women, 85\% were minorities, with mean age of $65 \pm 8.2$ years and BMI of $37.3 \pm 4.6 \mathrm{~kg} / \mathrm{m} 2$, were enrolled in the study at baseline however only 17 women in the intervention (EXD) group and 9 women in the wait-list control (CON) group completed the study. Measurement: Dietary quality and nutrition risk were measured using the Dietary Screening Tool (DST), resilience was measured by the Resilience Scale, and physical resilience was examined using the Physical Resilience Scale. Intervention: Participants in the EXD group engaged in 12 weeks of Tai Chi, resistance training, and behaviorally-based diet education. The diet education was based off of the modified Dietary Approaches to Stop Hypertension (DASH) diet and led by a Registered Dietitian. Results: There was no change in dietary quality by group or time. However the EXD group had significantly higher dietary quality compared to the control group ( $\mathrm{p}=0.025$ ) at post intervention, although there was no difference in nutrition risk category. There was no change seen in overall resilience, however the EXD group improved physical resilience $(p=0.048)$. Conclusion: A community health outreach that involved Tai Chi, resistance training, and behaviorally-based diet education may promote higher dietary quality as well as improve physical resilience in obese older women.
\end{abstract}

Key words: Physical resilience, dietary quality, Tai Chi, resistance training, modified DASH diet.

\section{Introduction}

Close to $50 \%$ of older adults may not have an optimal nutrition status $(1,2)$. This is a concern as good nutrition not only reduces the risk of chronic disease but is also a critical component of therapeutic plans for chronic diseases (3-6). The Dietary Approach to Stop Hypertension (DASH) diet, was originally developed to manage hypertension and has since been shown to help improve diet quality and facilitate weight management in adults (7-13). Women in particular are at greater risk as they are more likely to be obese, have chronic conditions, such as cardiovascular disease (CVD) and diabetes, and have a greater rate of functional decline due to aging compared to men (14-17).

Research has demonstrated that Tai Chi and resistance

1. Department of Nutrition and Food Sciences, University of Rhode Island, Ranger Hall, Kingston, Rhode Island, 02881, United States; 2. Department of Kinesiology, University of Rhode Island, Independence Square II, Kingston, Rhode Island, 02881, United States; 3. Department of Communicative Disorders, University of Rhode Island, Fernwood, Kingston, Rhode Island, 02881, United States

Corresponding Author: I.E. Lofgren Department of Nutrition and Food Sciences, University of Rhode Island, Ranger Hall, Kingston, Rhode Island, 02881, United States, ingridlofgren@uri.edu training are effective in improving anthropometrics, physical function, as well as symptoms of CVD, and diabetes in overweight and obese women (7-11, 18-28). Studies have also shown that Tai Chi and resistance training are well-tolerated exercise modalities in older adults (29-31). The ability to recover or optimize function in the face of age-related losses or diseases which facilitates recovery and coping with daily physical challenges associated with aging and chronic illness is physical resilience (32). Physical resilience is a component of overall resilience which is when a person positively adapts and adjusts to a significant source of stress, trauma or challenge (33). It is speculated that physical resilience could be modified following a health promotion intervention although overall resilience may not.

However, to date, there is limited research examining behaviorally-based diet education combined with multiple modalities of physical activity intervention on diet quality and resilience in obese women, especially in minority populations. Furthermore, there are no studies, to our knowledge, that combine diet education with both Tai Chi and resistance training. Therefore, the primary 
aim of this study was to examine dietary quality in older obese women in response to Tai Chi, resistance training, and a behaviorally-based diet education intervention in obese older women. The exploratory aim was to study the effect of a Tai Chi, resistance training and behaviorally-based diet education intervention in older obese women on physical resilience.

\section{Materials and methods}

\section{Study Design}

This study was a 12-week, quasi-experimental translational study conducted at an urban, Rhode Island (Providence, RI) senior center. The intervention consisted of Tai Chi, resistance training and behaviorallybased diet education. The study was approved by the University of Rhode Island Institutional Review Board. The intervention and all measures performed in this study were taken at baseline and post-intervention.

\section{Subjects}

There were 33 eligible participants of whom the first 23 were assigned to the intervention group and the remaining 10 women were assigned to the control group, see Table 1 for eligibility criteria.

Table 1

\section{Eligibility Criteria}

\footnotetext{
Inclusion Criteria

a. Women, aged $50-80$ years;

b. BMI of 30.0 to $50.0 \mathrm{~kg} / \mathrm{m} 2$;

c. Not engaged in regular exercise program within the past 6 months

d. Post-menopausal by self-report.

Exclusion Criteria

a. Failure to provide informed consent;

b. Significant or suspected cognitive impairment;

c. Severe hearing loss, speech disorder, language barrier or visual impairment;

d. Progressive, degenerative neurologic disease;

e. Terminal illness with life expectancy of $<12$ months, as determined by a physician;

f. Severe pulmonary disease, uncontrolled diabetes, blood pressure, or anemia;

g. Medications not taken for $>3$ weeks, lipid lowering medications for $>6$ months

h. Major joint, vascular, abdominal, or thoracic surgery within 6 months i. Significant cardiovascular disease;

j. Inability to safely engage in mild to moderate exercise with muscular exertion
}

\section{Dietary Quality and Nutritional Risk Classification}

Participants completed the Dietary Screening Tool (DST) which can identify dietary patterns and nutritional risk and has been validated in older adults $(2,34)$. The total score of the DST ranges from 0-100 with 5 'bonus' points for dietary supplement use; the higher the score indicating healthier dietary patterns. Furthermore, the composite score of the DST is associated with three nutritional risk levels; $(<60)$ at risk, $(60-75)$ possible risk, and $(>75)$ not at risk.

\section{Overall Resilience and Physical Resilience}

Overall resilience was measured using the Resilience Scale developed by Wagnild and Young, which is a series of 25 questions that are answered on a Likert scale from 1 to 7; higher scores indicate greater resilience (33). Physical resilience was measured via the Physical Resilience Scale, which was developed and validated by Resnick et al. $(32,35)$. This 15 -item, validated questionnaire has the participant use a physical challenge they have experienced on which to base their answers; higher scores indicate greater physical resilience.

\section{Demographics}

Demographics such as age, education, race, and chronic diseases were reported on the past medical history questionnaire.

\section{Anthropometrics}

Following an overnight fast and voiding of the bladder, weight was measured to the nearest 0.25 pound via a medical beam scale (Webb City, MO, USA) and height was measured with a stadiometer (Webb City, MO, USA) to the nearest $0.25 \mathrm{~cm}$; both were measured in duplicate and averaged. Body mass index (BMI; kg/ $\mathrm{m} 2$ ) was calculated from the average height and weight after appropriate conversions. Body composition was measured in all participants via foot-to-foot bioelectrical impedance analysis device (Tanita BF-556, Arlington Heights, IL, United States). The waist to hip ratio $(\mathrm{W}: \mathrm{H})$ was calculated from the waist circumference measurement using a standard tape measure with a tensometer (Creative Health Products, Ann Arbor, MI) at the point of the iliac crest and hip circumference measurement at the broadest circumference of the hips above the gluteal fold. 


\section{Cognitive Function}

The Repeatable Battery for the Assessment of Neurological Status (RBANS) test measures attention, language, visuospatial/constructional abilities, and immediate and delayed memory in individuals aged 20-89 years; the higher the score signifies better cognitive function (36).

\section{Intervention}

The intervention (EXD) group participated in three 90-minute sessions per week over a 12-week period on non-consecutive days. All sessions contained approximately 45 minutes of Yang-style Tai Chi. Two sessions per week included 30-45 minutes resistance training for the major muscle groups based on American College of Sports Medicine recommendations for older adults (37) and once per week there was 45 minutes of behaviorally-based diet education led by a Registered Dietitian. During the behaviorally-based diet education sessions, participants were encouraged to adopt a modified DASH diet as previously described $(9,11)$.

\section{Wait-list Control}

The control (CON) group did not receive any intervention during the 12 week intervention period and were asked to maintain their normal lifestyle.

\section{Statistical Analysis}

It was determined that a sample size of 24 total participants would be adequate in detecting a difference in DST score resulting in an effect size of 0.77 with an alpha of 0.05 and this number was consistent with past interventions (8-11,18-20). Data were analyzed using SPSS for Windows (version 22.0, IBM Corp. Summers, NY). Significance was set at a $p$ value $<0.05$. Data were checked for normality and transformations have been applied whenever was appropriate or nonparametric tests have been used. Data were assessed for differences between groups and completer status. All further tests were performed on completers only.

Between-group differences at baseline were compared using t-test or Mann-Whitney $U$ tests for continuous variables, and Fisher's exact test for categorical variables. Within group differences were assessed using a paired t-test or Wilcoxon Signed Rank test for continuous variables and McNemar's test for categorical variables. A one-way repeated measures analysis of variance (ANOVA) was used to compare baseline to postintervention variables by group.

\section{Results}

Seventeen participants completed the EXD group and 9 completed the wait-list control group, see Figure 1. Table 2 presents participant characteristics. Participants, in this study were primarily non-white $(85 \%)$ women with a mean age of $65 \pm 8.1$ years, BMI of $38.1 \pm 4.6 \mathrm{~kg} /$ $\mathrm{m} 2$ and the majority with a high school degree or less. More than half of the women had heart disease and $42 \%$ had diabetes. Mean cognitive function was $88.1 \pm 19.9$ which is considered to be in the below average range (36, 38, 39) Attendance was $67.5 \%$ for the overall intervention and $69.4 \%$ specifically for the dietary sessions. There was no difference in baseline variables between groups $(p>0.05)$ as well as was no difference in baseline variables in those who completed the study compared to those who did not.

Figure 1

Study Flow Chart

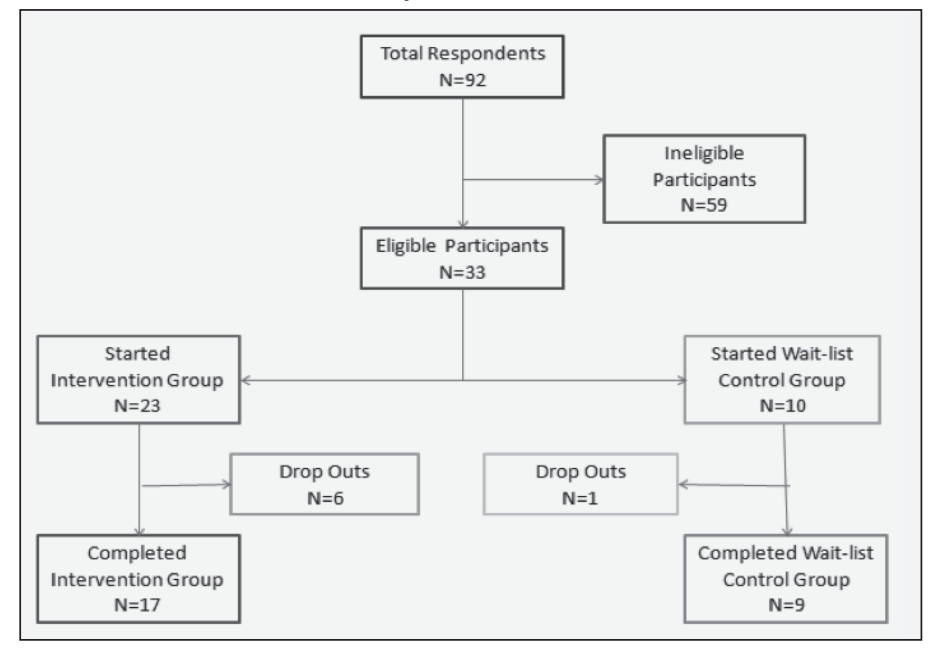

There was no significant effect for dietary quality in terms of group and time $(p=0.078)$, although the proportion of variance that dietary quality is explained by the intervention was considered a large effect (partial eta2 $=0.147)$. At post-intervention, the EXD group had significantly higher dietary quality $(66.5 \pm 10.2)$ compared to the CON group (54.4 $\pm 12.8, \mathrm{p}=0.025)$; see Figure 2 .

There were $87.5 \%$ of women at baseline who were at or possibly at nutrition risk. There was no significant difference in nutrition risk between groups at either baseline or post-intervention or within groups from baseline to post-intervention; see Figure 3 and 4 . However, five participants $(33.3 \%)$ in the EXD group improved their nutrition risk category, while no one in the CON group improved their nutrition risk category.

There was a significant group by time effect for physical resilience; with participants in the EXD group increasing their physical resilience from baseline to post intervention while those in the CON group decreased their physical resilience $(p=0.048)$. A large part of the variance of physical resilience was explained by the 
Table 2

Participant Characteristics

\begin{tabular}{|c|c|c|c|}
\hline & All $(n=26)$ & Intervention $(n=17)$ & Control $(n=9)$ \\
\hline Age (years) & $65 \pm 8.1$ & $65 \pm 8.1$ & $66 \pm 8.6$ \\
\hline Body Fat $(\%)$ & $49.7 \pm 3.1$ & $49.9 \pm 3.3$ & $49.7 \pm 2.9$ \\
\hline BMI $\left(\mathrm{kg} / \mathrm{m}^{2}\right)$ & $38.1 \pm 4.6$ & $38.8 \pm 5.1$ & $36.6 \pm 3.4$ \\
\hline \multicolumn{4}{|l|}{ Race $\%(n)$} \\
\hline Caucasian (non-Hispanic) & $15(4)$ & $24(4)$ & $0(0)$ \\
\hline Not Caucasian & $85(22)$ & $76(13)$ & $100(9)$ \\
\hline \multicolumn{4}{|l|}{ Education \%(n) } \\
\hline$\leq$ High School degree & $58(15)$ & $59(10)$ & $56(5)$ \\
\hline Some or college degree & $34(9)$ & $35(6)$ & $33(3)$ \\
\hline$>$ College degree & $8(2)$ & $6(1)$ & $11(1)$ \\
\hline CVD \%(n) & $69(18)$ & $65(11)$ & $78(7)$ \\
\hline Diabetes \%(n) & $42(11)$ & $35(6)$ & $56(5)$ \\
\hline Resilience & $154.9 \pm 19.8$ & $155.5 \pm 16.2$ & $153.7 \pm 26.0$ \\
\hline Activity Index a & $26.6 \pm 15.1^{¥}$ & $24.6 \pm 10.8$ & $30.1 \pm 22.1^{¥}$ \\
\hline Cognitive Function $b$ & $88.1 \pm 19.9$ & $86.7 \pm 18.6$ & $90.7 \pm 23.2^{¥}$ \\
\hline
\end{tabular}

a. Yale Physical Activity Scale questionnaire b. Repeatable Battery for the Assessment of Neurological Status test; ¥. Missing data

intervention given a large effect size (partial eta2 $=0.166$ ), see Figure 5. At post intervention, participants in the EXD group had significantly higher physical resilience (13.8 \pm 1.0$)$ compared to those in the control group (12.1 $\pm 2.5, \mathrm{p}=0.032$ ). A greater change in physical resilience was measured for participants in the EXD group who increased their physical resilience score $(0.2 \pm 1.1)$ while participants in the CON group's physical resilience score decreased $(-1.0 \pm 1.5, p=0.025)$. There was no change in overall resilience either between or within groups and overall resilience was not impacted by the intervention $(p=0.835$, partial eta $2=0.002)$.

Figure 2

Dietary Quality

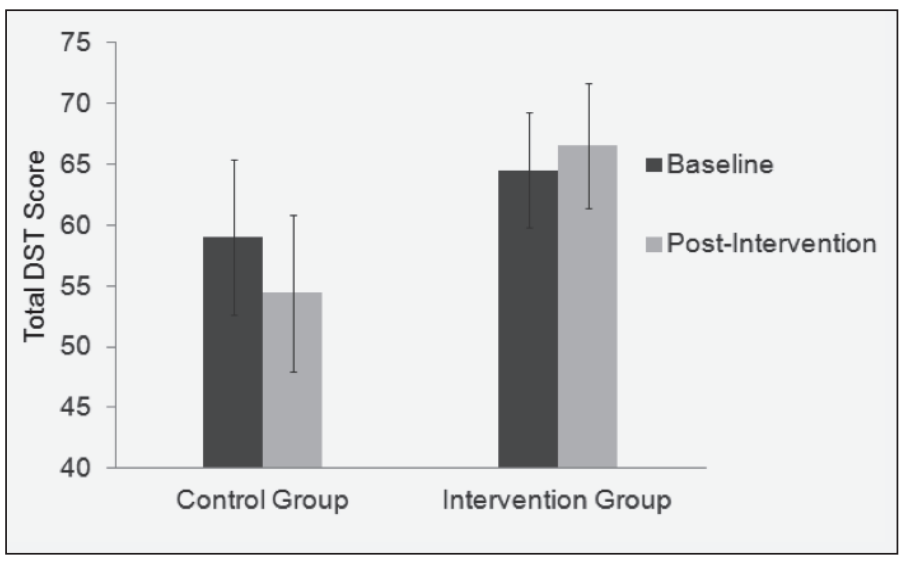

Depicts the differences (non-significant) in dietary quality in the CON group and EXD group at baseline and post-intervention.
Figure 3

Nutrition Risk in Control Group

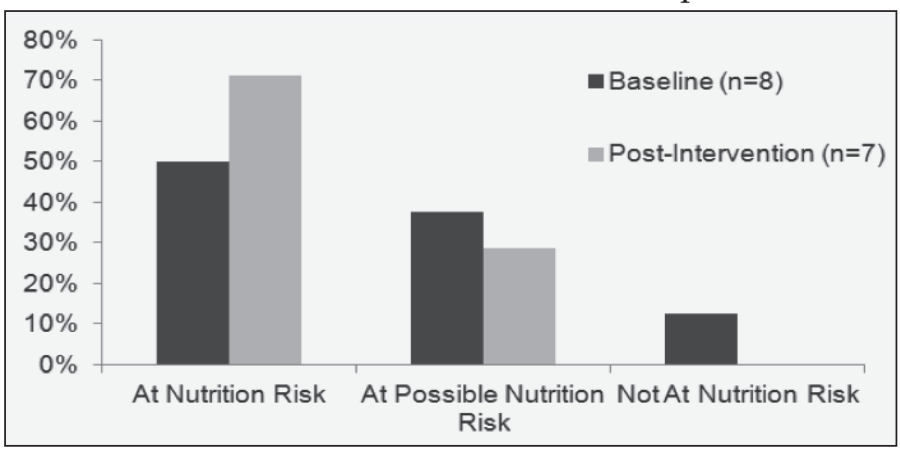

Depicts the percentage of participants in the CON group who were at nutrition risk, at possible nutrition risk, and not at nutrition risk at baseline and postintervention.

Figure 4

Nutrition Risk in Intervention Group

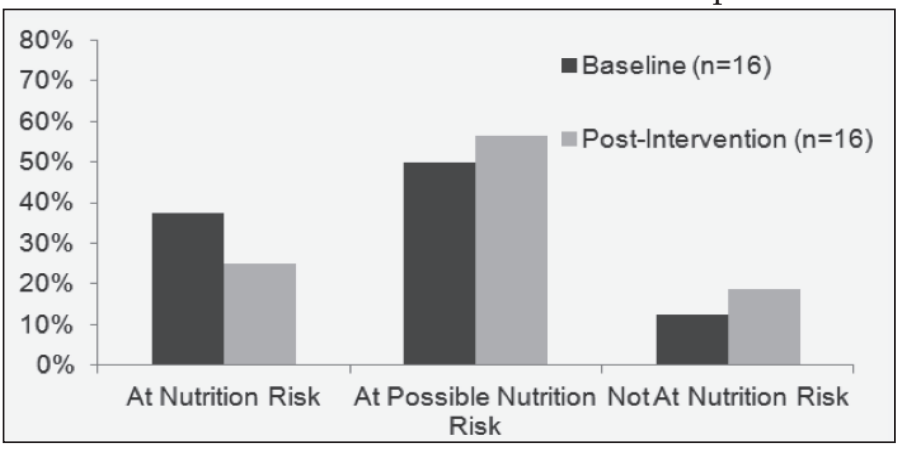

Depicts the percentage of participants in the EXD group who were at nutrition risk, at possible nutrition risk, and not at nutrition risk at baseline and postintervention. 
Figure 5

Physical Resilience

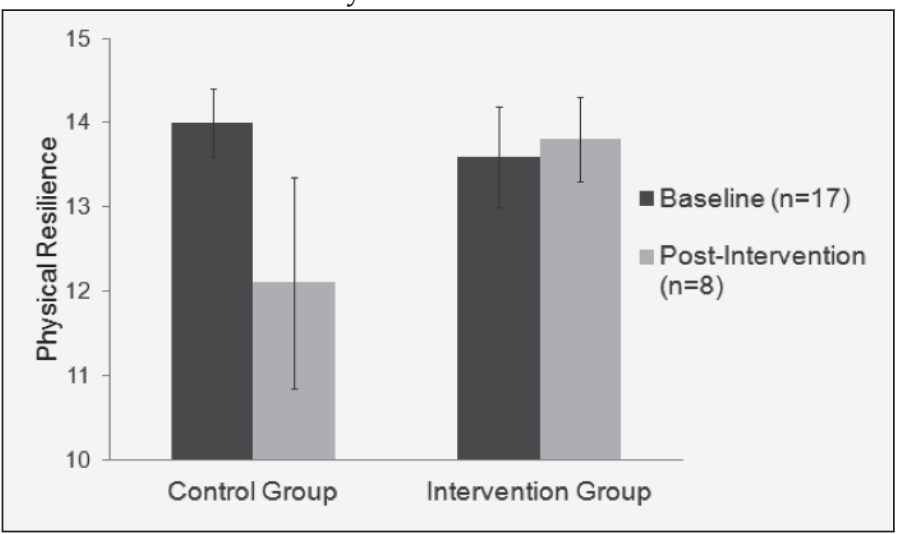

Depicts the mean physical resilience scores in both the CON and EXD group at baseline and post-intervention.

\section{Discussion}

The novel finding of this research is a trend indicating that 12-weeks of diet education combined with multiple modalities of physical activity may improve dietary quality along with other health outcomes in obese, mostly minority, older women. Furthermore, results from this study show that physical resilience can change in this at-risk population with a community health intervention.

There was no significant effect of time and group in diet quality in this study, although there was a large effect size (partial eta $2=0.147$ ), suggesting that with a larger sample size, the intervention could significantly improve dietary quality. Another study, with 16 weeks of behaviorally-based diet and Tai Chi education found that the intervention group significantly improved their dietary quality from baseline to post intervention in obese older women (9). Similarly, an 8-week community intervention in overweight and obese older adults resulted in a significant improvement in dietary quality from baseline to post-intervention (7). The fact that dietary quality was not statistically significant in the EXD group following the intervention, is likely a multifactorial issue. Unlike the studies previously mentioned, the majority of participants were minorities and had less education. Additionally, the mean cognitive function of the participants suggests that they may have had mild cognitive impairment $(36,38,39)$. This could partially explain the lack of a significant improvement in dietary quality in this current study. However, regardless of improvements in dietary quality in studies mentioned, the mean dietary quality scores suggests that participants are at or possibly at nutrition risk. This indicates that overall dietary quality needs to improve for older adults to be classified as not at nutrition risk. Further research is needed to explore improvements in dietary quality to a level that is classified as not at nutrition risk following behaviorally based diet education coupled with multiple modalities of exercise, particularly in lower educated minority obese women.

There was no difference in proportion of participants in nutrition risk categories between groups nor was there a change in nutrition risk over time in either group, $87.5 \%$ of completers were at nutrition risk as classified by DST score and only 5 participants increased their nutrition risk category at post intervention, all in the intervention group. This is of particular concern as individuals with good nutrition status have better overall health, decreased chronic disease, improved quality of life, increased life expectancy, better functional ability, and decreased disability (3-6).

Resilience is crucial for successful aging as it involves an individual's ability to adjust and adapt, which is particularly important as older adults often experience a loss of a spouse, other family members or close friend, an event that negatively impacts independence, or a general decline in physical health (40-41). However, there is controversy as to whether resilience is static (a trait) or dynamic (a state) (42). This debate partially hinges on whether personality can change. Research indicates that personality changes more drastically in younger years and becomes more stable with advancing age, which may influence how a person acutely and chronically deals with adverse life events $(43,44)$. Research also indicates that personality traits can change in adulthood, although the change is less pronounced compared to the change that occurs during younger years $(43,45)$. This study found the Tai Chi, resistance training, and behaviorally-based diet education has a beneficial effect in improving physical resilience, which is a specific type of resilience that emphasizes the physical aspect of resiliency, suggesting physical resilience may be dynamic and thus more of a state. However, there was no change in overall resilience following the intervention suggesting that either this intervention did not effectively target a change in overall resilience or that overall resilience may be a more of a trait.

There were several strengths of this study. The first is that the intervention examined multiple modalities of physical activity along with diet education; the combination of Tai Chi, resistance training, and diet education has not been studied before to our knowledge. Second, this study also had a large proportion of minority women, which is a strength as more research is needed on diet education and physical activity interventions in older minority women (46). Thirdly, the attendance rate was high given the large minority population. A systematic review found that the mean attendance rate for African Americans participating in nutrition and physical activity interventions was 58\% (47) which is lower than this study's attendance rate. The fourth strength is that this study used validated questionnaires and the intervention followed guidelines that have been shown to be effective in measuring targeted outcomes. Lastly, no studies, to our knowledge, have examined the impact of a lifestyle intervention on physical resilience. 
There remain some limitations to the current study that should be addressed in future research. First, assignment to the groups was non-randomized as it was a translational study designed to benefit participants. Despite this, the groups were very similar with regard to baseline characteristics. Second, the sample size was small, however the sample size is similar to previous studies and did show large effect sizes (8-11, 18-20). Third, individual intervention effects were not tested, but interventions that include diet education paired with Tai Chi or resistance training have been shown to significantly improve a host of health outcomes (7-11, 18-21).

\section{Conclusion}

The results from this study indicate a trend that a behaviorally-based diet education combined with Tai Chi and resistance training may positively impact dietary quality in obese older women in an urban setting. Furthermore, 12 weeks of Tai Chi, resistance training, behaviorally-based diet education beneficially improves physical resilience suggesting that physical resilience is dynamic. These results provide preliminary evidence that physical resilience is a state and therefore, amenable to change. Future research is needed to explore the effects of Tai Chi, resistance training, and behaviorally-based diet education with a larger sample of older women in this population on dietary quality and resilience to determine if overall resilience can change as well as physical resilience, particularly in obese older minorities.

Acknowledgement: We would like to thank Allison Picard for her assistance with study management. Supported by College of Environment and Life Sciences Community Access to Research and Extension Services (CELS CARES) grant, USDA

Ethical Standards: No ethics is required.

Conflicts of Interest: None

\section{References}

1. World Health Organisation. 2010a. Definition of an older or elderly 1. Soini $\mathrm{H}$, Routasalo $\mathrm{P}$, Lauri S. Nutrition in patients receiving home care in Finland: tackling the multifactorial problem. J Gerontol Nurs 2006;32(4):12-17

2. Bailey RL, Miller PE, Mitchell DC, Hartman TJ, Lawrence FR, Sempos CT, Simicklas-Weight H. Dietary screening tool identifies nutritional risk in older adults. Am J Clin Nutr 2009;90 (1):177-183. doi:10.3945/ajen.2008.27268

3. Sánchez García E, Montero Errasquín B, Sánchez Castellano C, Cruz-Jentoft AJ. Importance of nutritional support in older people. Nestle Nutr Inst Workshop Ser 2012;72:101-108. doi:10.1159/000339998

4. Woo J. Nutritional strategies for successful aging. Med Clin North Am 2011;95 (3):477-493, ix-x. doi:10.1016/j.mcna.2011.02.009

5. Gopinath B, Joanna Russell, Victoria M. Flood, George Burlutsky, Paul Mitchell. Adherence to Dietary Guidelines Positively Affects Quality of Life and Functional Status of Older Adults. J Acad Nutr Diet 2013;114(2):220-229. doi:10.1016/i.jand.2013.09.001

6. Fine JT, Colditz GA, Coakley EH, Moseley G, Manson JE, Willett WC, Kawachi I. A prospective study of weight change and health-related quality of life in women. JAMA 1999;282 (22):2136-2142. doi:10.1001/ jama.282.22.2136

7. Cottell KE, Dorfman LR, Straight CR, Delmonico MJ, Lofgren IE. The effects of diet education plus light resistance training on coronary heart disease risk factors in community-dwelling older adults. J Nutr Health Aging 2011;15(9):762-767. doi:10.1007/s12603-011-0099-0
8. Valente EA, Sheehy ME, Avila JJ, Gutierres JA, Delmonico MJ, Lofgren IE. The effect of the addition of resistance training to a dietary education intervention on apolipoproteins and diet quality in overweight and obese older adults. Clin Interv Aging 2011;6:235-241. doi:10.2147/CIA.S23583

9. Taetzsch A, Lobuono D, Beebe N, Letendre J, Bekke J, Delmonico M, Xu F, Lofgren I. The Effects of Behaviorally-Based Diet Education and Tai-Chi on Dietary Intake and Resiliency in Community Dwelling Older Adults. FASEB 2013;27:245.243. doi: 10.1096/fj. 1530-6860

10. Katkowski LA, Benson MC, Magnanti S, Lofgren IE, Xu F, Delmonico MJ. Additive effect of tai chi during dietary weight loss on physical function and body composition in obese older women. JARCP 2013;2(1):78-85

11. Beebe N, Magnanti S, Katkowski L, Benson M, Xu F, Delmonico MJ, Lofgren IE. Effects of the addition of $t^{\prime}$ ai chi to a dietary weight loss program on lipoprotein atherogenicity in obese older women. J Altern Complement Med 2013;19 (9):759-766. doi:10.1089/acm.2012.0531

12. Appel LJ, Moore TJ, Obarzanek E, Vollmer WM, Svetkey LP, Sacks FM, Bray GA, Vogt TM, Cutler JA, Windhauser MM, Lin PH, Karanja N. A clinical trial of the effects of dietary patterns on blood pressure. DASH Collaborative Research Group. NEJM 1997;336 (16):1117-1124. doi:10.1056/ nejm199704173361601

13. National Heart, Lung, and Blood Institute. Your Guide to Lowering your Blood Pressure with DASH. National Institutes of Health, 2006

14. Flegal KM, Carroll MD, Ogden CL, Johnson CL. Prevalence and trends in obesity among US adults, 1999-2000. JAMA 2002;288 (14):1723-1727

15. Crimmins EM, Saito Y, Reynolds SL. Further evidence on recent trends in the prevalence and incidence of disability among older Americans from two sources: the LSOA and the NHIS. J Gerontol Psychol Sci Soc Sci 1997;52 (2):S59-71

16. Anderson G. Chronic Care: Making the Case for Ongoing Care. Robert Wood Johnson Foundation, 2010

17. Mathus-Vliegen EM. Obesity and the elderly. J. Clin. Gastroenterol 2012;46 (7):533-544. doi:10.1097/MCG.0b013e31825692ce

18. Bekke J, Letendre J, Beebe N, Xu F, Lofgren I, Delmonico MJ. Effects of a Dietary and Tai Chi Intervention on Body Composition in Obese Older Women. FASEB 2013;27:1068.1062

19. Letendre JM, Bekke J, Beebe N, Lofgren IE, Xu F, Delmonico MJ. The Effects of Tai Chi plus behaviorally-based dietary weight loss on physical functioning in community-dwelling obese older women. FASEB 2013;27:712.711

20. Avila JJ. Effect of moderate intensity resistance training during weight loss on body composition and physical performance in overweight older adults. European journal of applied physiology 2010;109 (3):517-525. doi:10.1007/ s00421-010-1387-9

21. Straight CR, Dorfman LR, Cottell KE, Krol JM, Lofgren IE, Delmonico MJ. Effects of resistance training and dietary changes on physical function and body composition in overweight and obese older adults. J Phys Act Health 2012;9 (6):875-883

22. Wooten JS, Phillips MD, Mitchell JB, Patrizi R, Pleasant RN, Hein RM, Menzies RD, Barbee JJ. Resistance exercise and lipoproteins in postmenopausal women. Int J Sports Med 2011;32 (1):7-13. doi:10.1055/s-0030-1268008

23. Hornbuckle LM, Liu PY, Ilich JZ, Kim JS, Arjmandi BH, Panton LB. Effects of resistance training and walking on cardiovascular disease risk in AfricanAmerican women. Med Sci Sports Exerc 2012;44 (3):525-533. doi:10.1249/ MSS.0b013e31822e5a12

24. Christensen JR, Kristian Overgaard, Isabella G Carneiro, Andreas Holtermann, Karen Søgaard. Weight loss among female health care workers-a 1-year workplace based randomized controlled trial in the FINALE-health study. BMC Public Health 2012;12 (1):625. doi:10.1186/1471-2458-12-625

25. Lo H-M, Yeh CY, Chang SC, Sung HC, Smith GD. A Tai Chi exercise programme improved exercise behaviour and reduced blood pressure in outpatients with hypertension. IJNP 2012;18 (6):545-551. doi:10.1111/ ijn.12006

26. Park IS, Song R, Oh KO, So HY, Kim DS, Kim JI, Kim TS, Kim HL, Ahn SH. Managing cardiovascular risks with Tai $\mathrm{Chi}$ in people with coronary artery disease. J Adv Nurs 2010;66 (2):282-292. doi:10.1111/j.1365-2648.2009.05134.x

27. Chen SC, Ueng KC, Lee SH, Sun KT, Lee MC Effect of $t^{\prime}$ ai chi exercise on biochemical profiles and oxidative stress indicators in obese patients with type 2 diabetes. J Altern Complement Med 16 (11):1153-1159. doi:10.1089/ acm.2009.0560

28. Barbat-Artigas S, Filion ME, Dupontgand S, Karelis AD, Aubertin-Leheudre $\mathrm{M}$. Effects of tai chi training in dynapenic and nondynapenic postmenopausal women. Menopause 2011;18 (9):974-979. doi:10.1097/gme.0b013e3182127c89

29. Adler PA, Roberts BL (2006) The use of Tai Chi to improve health in older adults. Orthop Nurs 2006;25 (2):122-126

30. Yeh GY, Wood MJ, Lorell BH, Stevenson LW, Eisenberg DM, Wayne PM, Goldberger AL, Davis RB, Phillips RS. Effects of tai chi mind-body movement therapy on functional status and exercise capacity in patients with chronic heart failure: A randomized controlled trial. Am J Med 2004;117 (8):541-548. doi:DOI 10.1016/j.amjmed.2004.04.016

31. Roth SM, Ferrell RF, Hurley BF. Strength training for the prevention and treatment of sarcopenia. J Nutr Health Aging 2000;4 (3):143-155 
32. Resnick B, Galik E, Dorsey S, Scheve A, Gutkin S. Reliability and validity testing of the physical resilience measure. Gerontologist 2011;51 (5):643-652. doi:10.1093/geront/gnr016

33. Wagnild GM, Young HM. Development and Psychometric Evaluation of the Resilience Scale. J Nurs Meas 1993;1 (2):165-178

34. Bailey RL, Mitchell DC, Miller CK, Still CD, Jensen GL, Tucker KL, SmiciklasWright H. A Dietary Screening Questionnaire Identifies Dietary Patterns in Older Adults. J Nutr 2007;137 (2):421-426

35. Resnick BA, Inguito PL. The Resilience Scale: Psychometric Properties and Clinical Applicability in Older Adults. Arch Psychiatr Nurs 2011;25 (1):11-20. doi:10.1016/j.apnu.2010.05.001

36. Randolph C, Tierney MC, Mohr E, Chase TN. The Repeatable Battery for the Assessment of Neuropsychological Status (RBANS): Preliminary Clinical Validity. J Clin Exp Neuropsych 1998;20 (3):310-319. doi:10.1076/ jcen.20.3.310.823

37. American College of Sports Medicine WJ. American College of Sports Medicine position stand. Exercise and physical activity for older adults. Med Sci Sports Exerc 2009;41 (7):1510-1530. doi:10.1249/MSS.0b013e3181a0c95c

38. Duff K, Patterson D, Schoenberg MR, Mold J, Scott JG, Adams RL. Ageand education-corrected independent normative data for the RBANS in a community dwelling elderly sample. Clin Neuropsychol 2003;17(3):351-366

39. Duff K, Hoson VL, Beglinger LJ, O'Bryant SE. Diagnostic Accuracy of the RBANS in Mild Cognitive Impairment: Limitations on Assessing Milder Impairments. Arch Clin Neuropsych 2010;25(5),429-441. doi:10.1093/arclin/ acq045

40. Harris PB. Another wrinkle in the debate about successful aging: the undervalued concept of resilience and the lived experience of dementia. Int J Aging Hum Dev 2008;67 (1):43-61. doi:10.1177/1471301212442583

41. Wells M, Bergeman CS, Boker SM. Resilience in rural communitydwelling older adults. J rural health 2009;25 (4):415-419. doi:10.1111/j.17480361.2009.00253.x

42. Ong AD, Bergman CS, Boker SM. Resilience comes of age: defining features in later adulthood. J Pers 2009;77 (6):1777-1804. doi:10.1111/j.14676494.2009.00600.x

43. Roberts BW, DelVecchio W.F. The rank-order consistency of personality traits from childhood to old age: a quantitative review of longitudinal studies. Psychol Bull 2000;126 (1):3-25. doi: 10.1037/0033-2909.126.1.3

44. McCrae RR, Costa PT. The Stability of Personality: Observations and Evaluations. Curr Dir Psychol Sci 1994;3 (6):173-175. doi:10.2307/20182303

45. Pervin LA. A Critical Analysis of Current Trait Theory. Psychol Inq 1994;5 (2):103-113. doi:10.1207/s15327965pli0502_1

46. Moreno-John G, Gachie A, Fleming CM, Nápoles-Springer A, Mutran E Manson SM, Pérez-Stable EJ. (Ethnic minority older adults participating in clinical research: developing trust. J Aging Health 2004;16 (5):93S-123S. doi: $10.1177 / 0898264304268151$

47. Lemacks J, Wells BA, Ilich JZ, Ralston PA. Interventions for improving nutrition and physical activity behaviors in adult African American populations: a systematic review, January 2000 through December 2011. Prev Chronic Dis 2013;10:E99. doi:10.5888/pcd10.120256 